\title{
Aerobic exercise attenuates pulmonary injury induced by exposure to cigarette smoke
}

\author{
A.C. Toledo*, R.M. Magalhaes*, D.C. Hizume*,", R.P. Vieira*,", P.J.C. Biselli*, \\ H.T. Moriya ${ }^{+}$, T. Mauad ${ }^{\S}$, F.D.T.Q.S. Lopes* and M.A. Martins*
}

ABSTRACT: It has recently been suggested that regular exercise reduces lung function decline and risk of chronic obstructive pulmonary disease (COPD) among active smokers; however, the mechanisms involved in this effect remain poorly understood.

The present study evaluated the effects of regular exercise training in an experimental mouse model of chronic cigarette smoke exposure.

Male C57BL/6 mice were divided into four groups (control, exercise, smoke and smoke+exercise). For 24 weeks, we measured respiratory mechanics, mean linear intercept, inflammatory cells and reactive oxygen species (ROS) in bronchoalveolar lavage (BAL) fluid, collagen deposition in alveolar walls, and the expression of antioxidant enzymes, matrix metalloproteinase 9, tissue inhibitor of metalloproteinase (TIMP)1, interleukin (IL)-10 and 8-isoprostane in alveolar walls.

Exercise attenuated the decrease in pulmonary elastance $(p<0.01)$ and the increase in mean linear intercept $(p=0.003)$ induced by cigarette smoke exposure. Exercise substantially inhibited the increase in ROS in BAL fluid and 8-isoprostane expression in lung tissue induced by cigarette smoke. In addition, exercise significantly inhibited the decreases in IL-10, TIMP1 and CuZn superoxide dismutase induced by exposure to cigarette smoke. Exercise also increased the number of cells expressing glutathione peroxidase.

Our results suggest that regular aerobic physical training of moderate intensity attenuates the development of pulmonary disease induced by cigarette smoke exposure.

KEYWORDS: Aerobic exercise, chronic obstructive pulmonary disease, cigarette smoke, emphysema, oxidative stress

lthough the majority $(80 \%)$ of patients with chronic obstructive pulmonary disease (COPD) are cigarette smokers, only a small proportion (15-20\%) of cigarette smokers develop COPD [1], suggesting that there are genetic and/or environmental susceptibility factors involved in the development of this syndrome. Recently, GARCIAAYMERICH et al. [2] observed that active smokers who practised moderate or high levels of regular physical activity had a reduced risk of developing COPD compared with the low physical activity group. Previous studies have shown that physical exercise increases the resistance against oxidative stress in skeletal muscle [3]. Some epidemiological studies suggest that regular physical exercise reduces the incidence of diseases associated with oxidative stress [4]. This phenomenon is considered a result of adaptation induced by exercise. This process involves the activation of the antioxidant system and redox reactions, and it influences transcription and, thus, gene expression and assembly of proteins [5].

COPD is an important cause of morbidity, mortality and healthcare costs worldwide [6]. Its prevalence is around $9-10 \%$ among individuals aged $>40$ yrs but can reach up to $20 \%$ in some areas of the world [1]. Tobacco smoking is the most important risk factor for COPD. Although the pathogenesis of COPD remains incompletely understood, the importance of oxidative stress in lungs and systemic circulation is well established [7]. In the gas phase of cigarette smoke there are high concentrations of many oxidants/free radicals ( $>10^{15}$ molecules per puff) [8]. In addition, there are also endogenous sources of reactive oxygen species (ROS), such as those produced by activated leukocytes recruited to the lungs in response to cigarette smoke. ROS derived from both cigarette smoke and endogenous sources can damage lipids, proteins and nucleic acids and,
AFFILIATIONS

Depts of *Medicine (LIM-20) and SPathology (LIM-05), School of Medicine and

+Biomedical Engineering Laboratory, Escola Politecnica, University of Sao

Paulo, Sao Paulo and

\#Dept of Physical Therapy, State University of Santa Catarina,

Florianopolis, Brazil.

"Dept of Pneumology, University Hospital Freiburg, Freiburg, Germany.

CORRESPONDENCE

M.A. Martins

Faculdade de Medicina da Universidade de São Paulo Av. Dr. Arnaldo 455 sala 1210

01256-903

São Paulo

Brazil

E-mail: mmartins@usp.br

Received:

Jan 092011

Accepted after revision:

June 132011

First published online:

June 232011

Online ISSN 1399-3003 
in some cases, induce the formation of more stable reactive molecules that can prolong the oxidant-mediated damage [9].

The purpose of our study was to evaluate the effects of regular physical training during the establishment of pulmonary alterations induced by chronic ( 24 weeks') exposure to cigarette smoke in mice. Our hypothesis was that aerobic conditioning could attenuate the development of pulmonary disease induced by cigarette smoke exposure. Since there is strong evidence that an impairment in oxidant-antioxidant balance is important in the pathogenesis of pulmonary effects of cigarette smoke, we evaluated the effects of cigarette smoke, exercise training and both on the pulmonary oxidant-antioxidant balance in the lungs.

\section{METHODS}

\section{Experimental groups}

In all experiments, the Helsinki convention for use and care of animals was followed. Our protocol was approved by the ethical committee of the School of Medicine of the University of Sao Paulo (Sao Paulo, Brazil). Male C57BL/6 mice (6-8 weeks old) were divided at random into four groups ( $n=25-32$ per group): 1) control; 2) exercise (submitted to treadmill training); 3 ) smoke (exposed to cigarette smoke) and 4) smoke+exercise (submitted to treadmill training and exposed to cigarette smoke). Animals were submitted to exercise training and exposed to cigarette smoke for 60 and $30 \mathrm{~min} \cdot \mathrm{day}^{-1}$, respectively, 5 days $\cdot$ week $^{-1}$ for 24 weeks. The smoke+exercise group was initially submitted to the exercise protocol, the mice were allowed to rest for $\geqslant 60 \mathrm{~min}$, then the exposure to cigarette smoke was started.

\section{Cigarette smoke exposure protocol}

Smoke exposure was performed in an inhalation chamber (28 L), with two inlets (for air and smoke), one outlet and a fan, for enhancing air and smoke mixture inside the box. Synthetic airflow in the first inlet was set at $2 \mathrm{~L} \cdot \mathrm{min}^{-1}$, and the second inlet received a synthetic airflow that passed through a Venturi System connected to a lit cigarette, suctioning the cigarette smoke and conducting it inside the box. The airflow rate in this second inlet could be changed, providing more or less smoke mixture to the box. After several measurements of carbon monoxide (CO) concentration inside the box, we set this flow rate at $1.5 \mathrm{~L} \cdot \mathrm{min}^{-1}$, which produced CO levels ranging from 250 to $350 \mathrm{ppm}$. Carboxyhaemoglobin concentration in smoke-exposed mice was kept at $10 \%( \pm 1.3 \%)$. Animals were exposed to $12( \pm 1)$ commercially filtered cigarettes per day $(0.8 \mathrm{mg}$ of nicotine, $10 \mathrm{mg}$ of tar and $10 \mathrm{mg}$ of $\mathrm{CO}$ per cigarette) and total particulate matter concentration $354.8 \pm 50.3 \mu \mathrm{g} \cdot \mathrm{m}^{-3} \cdot \mathrm{day}^{-1}$. Mice allocated to cigarette smoke exposure groups were kept in the box maintaining these CO levels for $30 \mathrm{~min} \cdot$ day $^{-1}, 5$ days $\cdot$ week $^{-1}$ for 24 weeks. Control mice were exposed to room air.

\section{Treadmill aerobic training and test}

Mice were initially adapted to the treadmill for 3 days $\left(15 \mathrm{~min} \cdot\right.$ day $^{-1}, 25 \%$ inclination and $\left.0.2 \mathrm{~km} \cdot \mathrm{h}^{-1}\right)$. After that, a maximal exercise capacity test was performed with a 5-min warm-up $\left(25 \%\right.$ inclination and $\left.0.2 \mathrm{~km} \cdot \mathrm{h}^{-1}\right)$ followed by an increase in treadmill speed $\left(0.1 \mathrm{~km} \cdot \mathrm{h}^{-1}\right.$ every $\left.2.5 \mathrm{~min}\right)$ until animal exhaustion, i.e. until they were not able to run even after 10 gentle mechanical stimuli [10]. The test was repeated four times: initially, and 8, 16 and 24 weeks after the beginning of the exposure to either cigarette smoke or room air. Body weight, speed and total test time reached for each mouse were registered. Maximal aerobic capacity $(100 \%)$ was established as the speed reached by each animal. Mice were trained at a moderate intensity of exercise ( $50 \%$ of maximal speed) for $60 \mathrm{~min} \cdot$ day $^{-1}$, 5 days $^{*}$ week $^{-1}$ for 24 weeks. Aerobic conditioning began on the same day as the beginning of cigarette smoke exposure and continued until the end of the 24th week. Both protocols were performed in the morning. The smoke+exercise group was initially submitted to the exercise protocol, then mice were allowed to rest for $\geqslant 60 \mathrm{~min}$ to allow respiratory frequency to return to basal (pre-exercise) values, then the exposure to cigarette smoke was started.

\section{Respiratory mechanics evaluation}

$24 \mathrm{~h}$ after the end of the exposure protocol, mice were anaesthetised, tracheostomised and mechanically ventilated $(n=10-15$ per group) using a FlexiVent ventilator (Scireq, Montreal, QC, Canada). Using the forced oscillatory technique and a constant phase model [11], airway resistance (Raw), tissue damping (G) and tissue elastance $(\mathrm{H})$ parameters were obtained. $\mathrm{G}$ and $\mathrm{H}$ were normalised to body weight [12].

\section{Total cells, macrophages and ROS measurement in bronchoalveolar lavage}

Immediately after lung mechanics measurements, mice were killed by exsanguination, and bronchoalveolar lavage (BAL) samples were collected after washing the lungs with $0.5 \mathrm{~mL}$ of sterile saline. This procedure was repeated three times. $400 \mu \mathrm{L}$ of cell suspension was added to $10 \mu \mathrm{L}$ of $5 \mathrm{mM}$ luminol (5-amino-2,3-dihydro-1,4-phthalazinedione; Sigma Chemical Co., St Louis, MO, USA) prepared in dimethyl sulfoxide (DMSO; Sigma Chemical Co.). ROS production was measured by a chemiluminescence assay method using luminol as the probe and a luminometer (MicroBeta TriLux, version 4.7; Perkin Elmer Life Sciences, Turku, Finland) for $15 \mathrm{~min}$ [13]. The results were expressed as number of photons per minute per $10^{4}$ cells per millilitre of BAL. The BAL fluid collected was centrifuged at $900 \times g$ for $8 \mathrm{~min}$ at $5^{\circ} \mathrm{C}$, and the cell pellet was resuspended in $1 \mathrm{~mL}$ of physiological saline. Total cells were counted using a Neubauer haemocytometer chamber (Carl Roth, Karlsruhe, Germany) and differential cells (300 cells $\cdot$ slide ${ }^{-1}$ ) were evaluated by microscopic examination of BAL samples prepared in cytocentrifuge slides, stained with Diff Quick (Medion Diagnostics, Dündingen, Switzerland) [14].

\section{Lung histology and immunohistochemistry}

Lungs were fixed at a constant pressure $\left(20 \mathrm{cmH}_{2} \mathrm{O}\right)$, and $5-\mu \mathrm{m}$ thick sections of lung tissue were stained with haematoxylin and eosin to measure mean linear intercept, an indicator of mean diameter of airspaces, as described previously [15]. Sirius red staining [16] was performed to evaluate collagen fibres by image analysis. Lung tissue was also immunostained with the following antibodies using the biotin-streptavidin peroxidase method: antiglutathione peroxidase (anti-GPx; goat polyclonal, 1:150), anti$\mathrm{CuZn}$ superoxide dismutase (anti-CuZnSOD; goat polyclonal, 1:400), anti-MnSOD (goat polyclonal, 1:200), anti-matrix metalloproteinase 9 (anti-MMP9; goat polyclonal, 1:400), anti-tissue inhibitor of metalloproteinase 1 (anti-TIMP1; rabbit polyclonal, 1:250), anti-interleukin (IL)-10 (rabbit polyclonal, 1:200), anti-extracellular SOD (anti-ECSOD; goat polyclonal, 1:400), anti-monocyte chemotactic protein 1 (anti-MCP1; goat polyclonal, 1:3,500), anti-tumour 
necrosis factor- $\alpha$ (anti-TNF- $\alpha$; goat polyclonal, 1:1,800), anti-IL-1 receptor antagonist (anti-IL-1ra; goat polyclonal, 1:500) (all Santa Cruz Biotechnology, Santa Cruz, CA, USA) and anti-8-isoprostane (goat polyclonal, 1:500; Oxford Biomedical Research, Rochester Hills, MI, USA). Secondary antibodies from the Vector ABC Kit Vectastain Anti-Rabbit or Vector ABC Kit Vectastain Anti-Goat, both diluted 1:500 (Vector Laboratories, Inc., Burlingame, CA, USA), were used for reactions. These secondary antibodies were also applied without primary antibody in positive and negative tissue samples, serving as controls. Positive cells immunostaining with GPx, CuZnSOD, MnSOD, MMP9, TIMP1, IL-10, MCP1, TNF- $\alpha$ and IL-1ra in the alveolar parenchyma were assessed using a 100-point grid with a known area $\left(62,500 \mu^{2}\right.$ at $\times 400$ magnification) attached to the microscope ocular. We counted the number of points hitting alveolar tissue in each field. The alveolar tissue area in each field was calculated according to the number of points hitting alveolar tissue, as a proportion of the total grid area. We then counted the number of positive cells within that alveolar tissue area and expressed the results as cells per $10^{6} \mu \mathrm{m}^{2}$ [17]. We also evaluated the volume proportion of 8-isoprostane- or ECSODpositive area expressed as the percentage of the positively immunostained area in relation to the total area of alveolar tissue. It should be clarified that the areas of airways and large vessels were excluded from the total area counted for analysis.

\section{Statistical analysis}

Statistical analysis was performed using Sigma Stat 10 software (Systat Software, Inc., San Jose, CA, USA). Comparisons among groups were carried out by ANOVA followed by the HolmSidak method for parametric data, or by ANOVA on ranks followed by Dunn's method for nonparametric data. Differences were considered significant at $\mathrm{p}<0.05$.

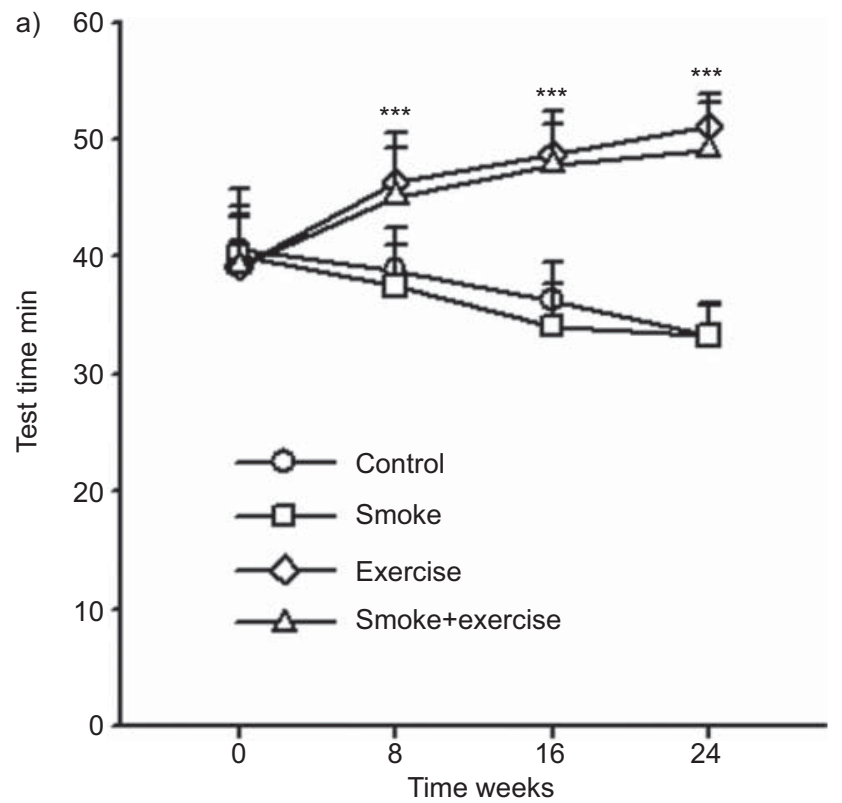

\section{RESULTS}

\section{Physical test: time and speed}

Figure 1 shows that after 8 weeks of training, the time that the mice could exercise on the treadmill at maximal speed was significantly greater in the exercise and smoke+exercise groups compared to the groups that were not trained (control and smoke; $p<0.001$ ). Similar results were observed after 16 and 24 weeks of training (fig. 1a). Exposure to cigarette smoke did not influence the results of the exercise tests. In fact, there were no significant differences between control and smoke groups or between exercise and smoke+exercise groups. Similar results were observed when maximal speed reached by the mice during the treadmill test was examined (fig. 1b).

\section{Respiratory mechanics evaluation}

Raw, G and H were computed (fig. 2). The values were normalised to body weight because the experimental groups that were trained presented greater body mass at the end of the experimental protocol $(\mathrm{p}<0.01$ compared with control and smoke groups; data not shown) [14]. We did not observe significant differences in Raw values when the four experimental groups were compared (fig. 2a). Exposure to cigarette smoke resulted in lower values of $G(p<0.01$ compared with the two groups that were not exposed to smoke; fig. $2 b$ ). Physical conditioning did not influence this response. In contrast, $\mathrm{H}$ values were lower in the group exposed to cigarette smoke for 24 weeks $(p<0.01$ compared with the other three groups; fig. 2c) and this decrease was not observed in the smoke+exercise group.

\section{Emphysema development and pulmonary parenchyma remodelling}

Exposure to cigarette smoke for 6 months resulted in an increase in airspace size and rupture of alveolar septa: hallmarks of

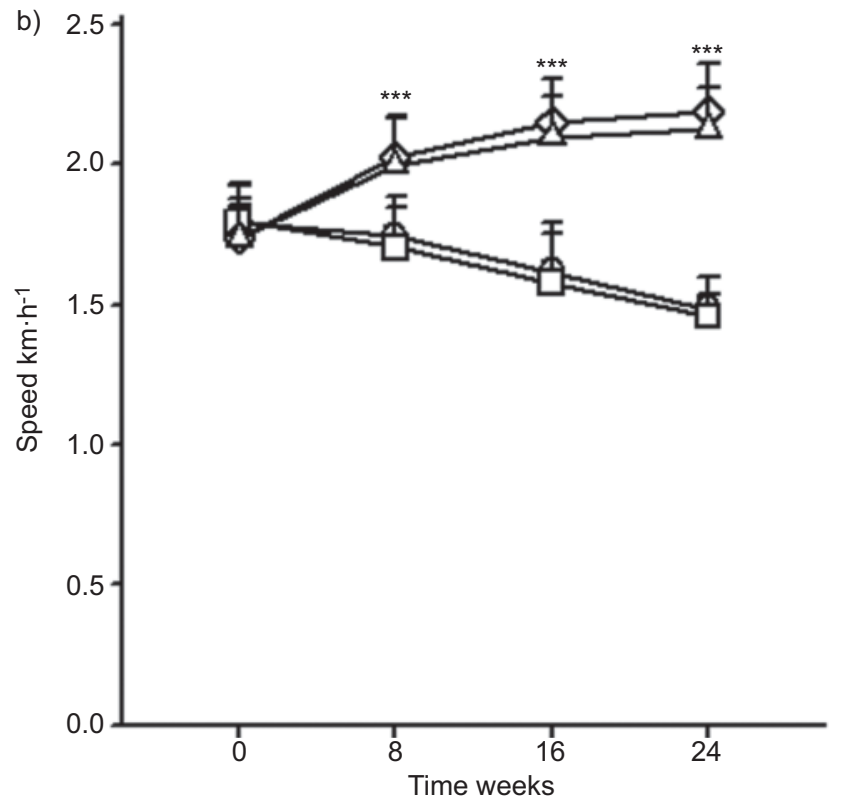

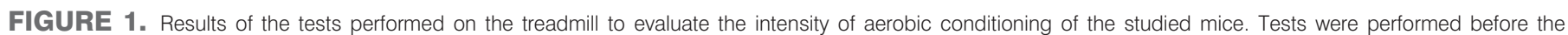

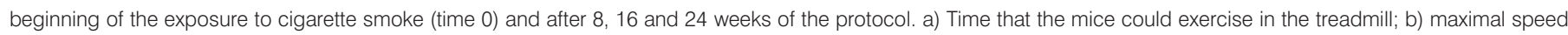

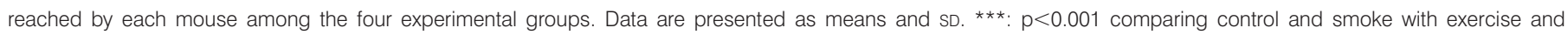
smoke+exercise. 

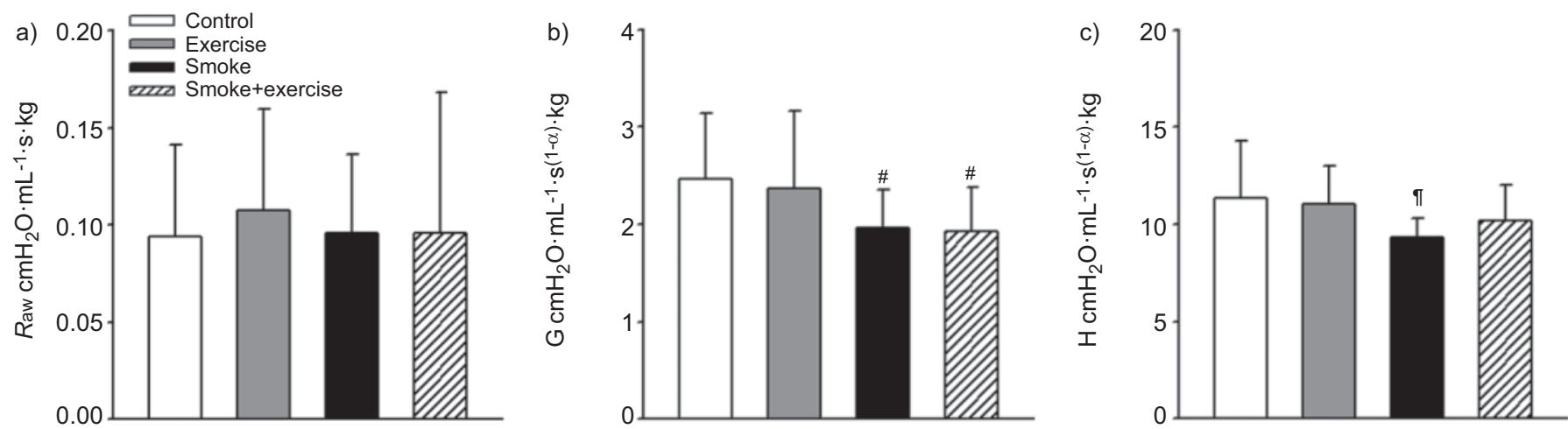

FIGURE 2. a) Airway resistance (Raw), b) tissue damping (tissue resistance; G) and c) pulmonary elastance (H) obtained in the four experimental groups, after 24 weeks of the experimental protocol. Data are presented as means and SD. The values were normalised to body weight. ${ }^{\#}: \mathrm{p}<0.01$ compared with the two groups that were not exposed to smoke; " $\mathrm{p}<0.01$ compared with the other three groups.

emphysema. We observed an increase in mean linear intercept in the two lung compartments in the group of mice exposed to cigarette smoke only (smoke group; $\mathrm{p}=0.003$ and $\mathrm{p}=0.002$ compared with the other three groups, for central and peripheral airspaces, respectively; fig. 3a-e). The group of mice exposed to cigarette smoke and submitted to the aerobic conditioning protocol (smoke+exercise group) did not show the increase in mean linear intercept compared with control and exercise groups.

Exposure to cigarette smoke resulted in the remodelling of the alveolar wall (fig. $3 \mathrm{f}-\mathrm{j}$ ). There was a significant increase in collagen fibres in the lungs of mice that were exposed to cigarette smoke $(p<0.001$ compared with the groups not exposed to smoke). Physical training did not influence this response to cigarette smoke.

\section{Leukocytes and ROS in BAL fluid}

Exposure to cigarette smoke resulted in an increase in the number of macrophages in BAL ( $p<0.01$ compared with the groups that were not exposed to smoke; fig. 4a). Exercise training did not influence this response. There were no significant differences in the numbers of neutrophils or lymphocytes in BAL among the four experimental groups (data not shown). Figure $4 \mathrm{~b}$ shows the concentrations of ROS in BAL. Exposure to cigarette smoke increased the production of ROS $(\mathrm{p}=0.029$ compared with control) and aerobic training significantly reduced ROS production $(\mathrm{p}=0.001$ compared with control). The group of mice that were submitted to both exercise and cigarette smoke showed values of ROS similar to the group that exercised but was not exposed to smoke ( $\mathrm{p}<0.001$ compared with smoke group).

\section{Immunohistochemical studies}

Exposure to cigarette smoke resulted in increase in the amount of 8-isoprostane in lung parenchyma, and this increase was not observed in the smoke+exercise group (fig. 5). In fact, expression levels of 8-isoprostane in lung parenchyma were significantly greater in the smoke group compared with the other three groups $(\mathrm{p}<0.01)$.

Exercise training resulted in an increase in the density of cells expressing GPx in alveolar walls $(\mathrm{p}<0.001$ for exercise versus control and smoke groups; fig. 6a-e). The smoke+exercise group showed intermediate numbers of cells expressing GPx $(\mathrm{p}<0.001$, $p=0.008$ and $p=0.046$ compared with exercise, smoke and control groups, respectively). Exposure to cigarette smoke reduced the density of cells in the alveolar walls positive for CuZnSOD, and exercise training inhibited this reduction $(p<0.01$ for the smoke group versus the other three groups; fig. $6 \mathrm{f}-\mathrm{j}$ ). Exposure to cigarette smoke and aerobic physical training did not change the density of alveolar wall cells positive for MnSOD and ECSOD (fig. 6k and 1).

Smoke exposure reduced the density of alveolar wall cells positive for TIMP1, and physical training inhibited this reduction ( $p<0.02$ for smoke versus the other three groups; fig. 7a-e). Smoke exposure and aerobic physical training did not significantly change the density of cells in alveolar walls positive for MMP9 (fig. 7f).

The smoke group had a lower density of IL-10-positive cells than both groups that exercised $(\mathrm{p}=0.013$ and $\mathrm{p}=0.009$ versus exercise and smoke+exercise groups, respectively; fig. 8a-e). Exposure to cigarette smoke increased the density of MCP1-positive cells in alveolar wall, and physical training inhibited this increase $(\mathrm{p}<0.01$ for smoke versus the other three groups; fig. $8 \mathrm{f}-\mathrm{j})$. There was a trend for a higher density of positive cells for TNF- $\alpha$ in the lungs of mice exposed to cigarette smoke $(p=0.053$; fig. $8 \mathrm{k}$ ), but no statistically significant differences in the expression of IL-1ra in the lungs of mice of the four experimental groups $(\mathrm{p}=0.09$; fig. 8l).

\section{DISCUSSION}

In this study, we showed that aerobic physical training of moderate intensity reduces oxidative stress and protects against emphysema development induced by cigarette smoke exposure in mice. Exercise training inhibited the increase in mean linear intercept and the decrease in pulmonary elastance induced by cigarette smoke but did not affect collagen fibre remodeling. In addition, we observed a reduction in the production of ROS in BAL, lower accumulation of 8-isoprosane in pulmonary tissue, and an increase in the expression of antioxidant enzymes GPx and CuZnSOD induced by exercise training. These results suggest that aerobic training has a protective effect on the imbalance between oxidants and antioxidants involved in the pathogenesis of cigarette smoke-induced pulmonary disease.

There are many previous studies evaluating the effects of exercise training or cigarette smoke exposure on the oxidant-antioxidant 


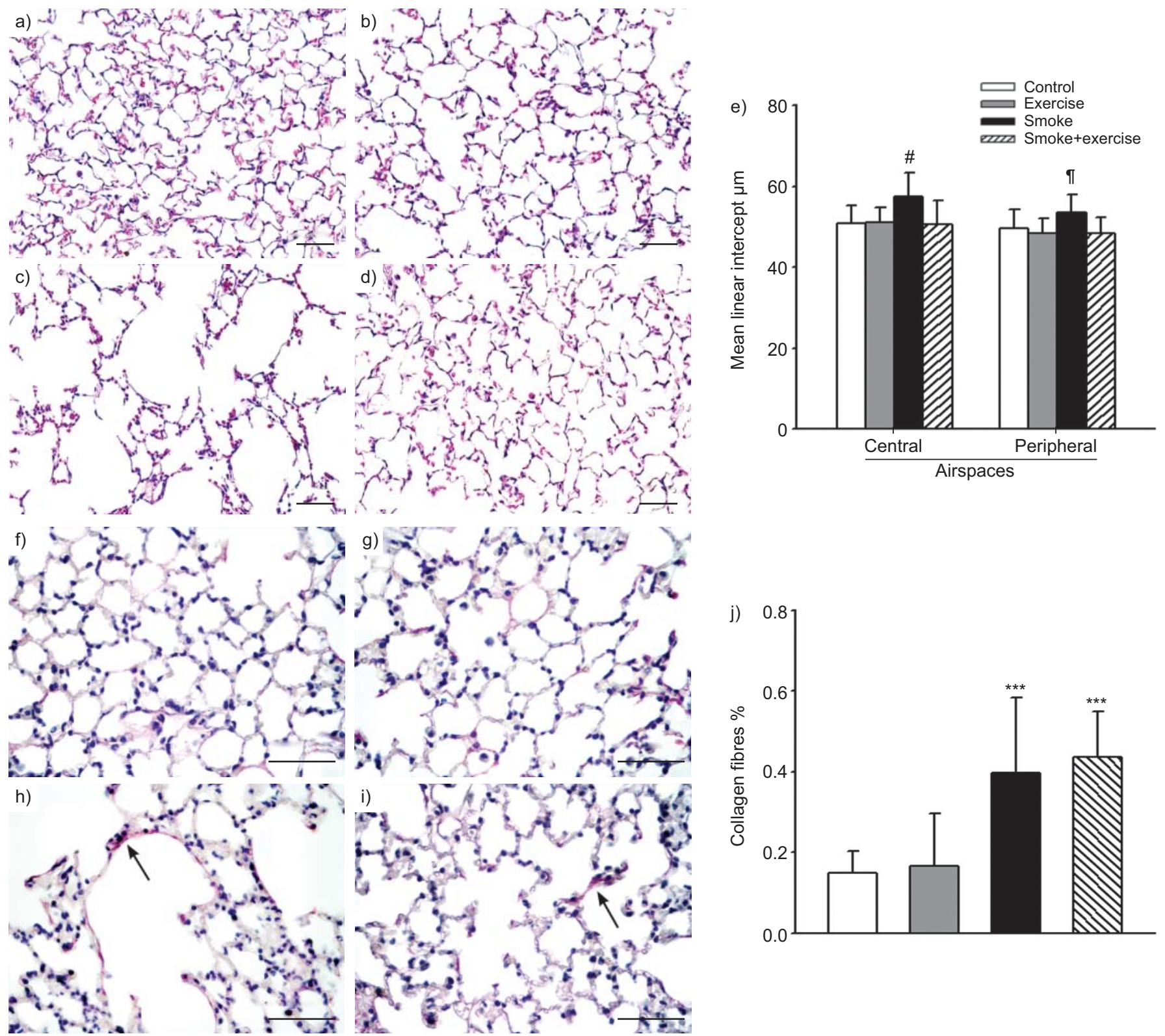

FIGURE 3. a--d) Representative photomicrographs of haematoxylin- and eosin-stained pulmonary parenchyma of a) control, b) exercise, c) smoke and d) smoke+exercise groups. e) Mean linear intercept measured in central and peripheral areas of the lungs. f-i) Representative photomicrographs of slices of lung parenchyma from the f) control, g) exercise, h) smoke and i) smoke+exercise groups, stained with Sirius red for collagen fibres (arrows). j) Percentage of the alveolar wall with collagen fibres. Scale bars: $50 \mu \mathrm{m}$. Data in e) and j) are presented as means and SD. ${ }^{\#}: p=0.003$ compared with the other three groups; ${ }^{\circ}: p=0.002$ compared with the other three groups; $* * *: p<0.001$ compared with the groups not exposed to cigarette smoke.

balance. However, only a few studies have aimed to evaluate the combined effects of exercise training and smoke. To our knowledge, our study was the first to evaluate the effects of exercise training on the development of emphysema during long-term (24 weeks') cigarette smoke exposure and to evaluate the possible mechanisms of the protective effects of long-term regular exercise, including the expression of enzymes involved in the oxidant-antioxidant balance in the lungs.

The experimental model used in our study shared several features with human COPD, such as exposure to cigarette smoke being a risk factor, a reduction in lung elastance and the development of pulmonary emphysema [18].
It is well established that several months of exposure to cigarette smoke are required for mice to develop pulmonary emphysema (usually in the order of 24 weeks) [18]. The susceptibility of mice to this disease is dependent on the mouse strain [19]. C57BL/6 mice exposed to cigarette smoke for 24 weeks show enlargement of airspaces and a pulmonary histology similar to human emphysema [20]. A previous study showed a partial improvement in pulmonary alterations in C57BL/6 mice exposed to cigarette smoke for 2 months and subjected to swimming [21].

Interestingly, in our study, mice exposed to cigarette smoke did not perform worse in the physical tests when compared with mice not exposed to smoke. This probably occurred 

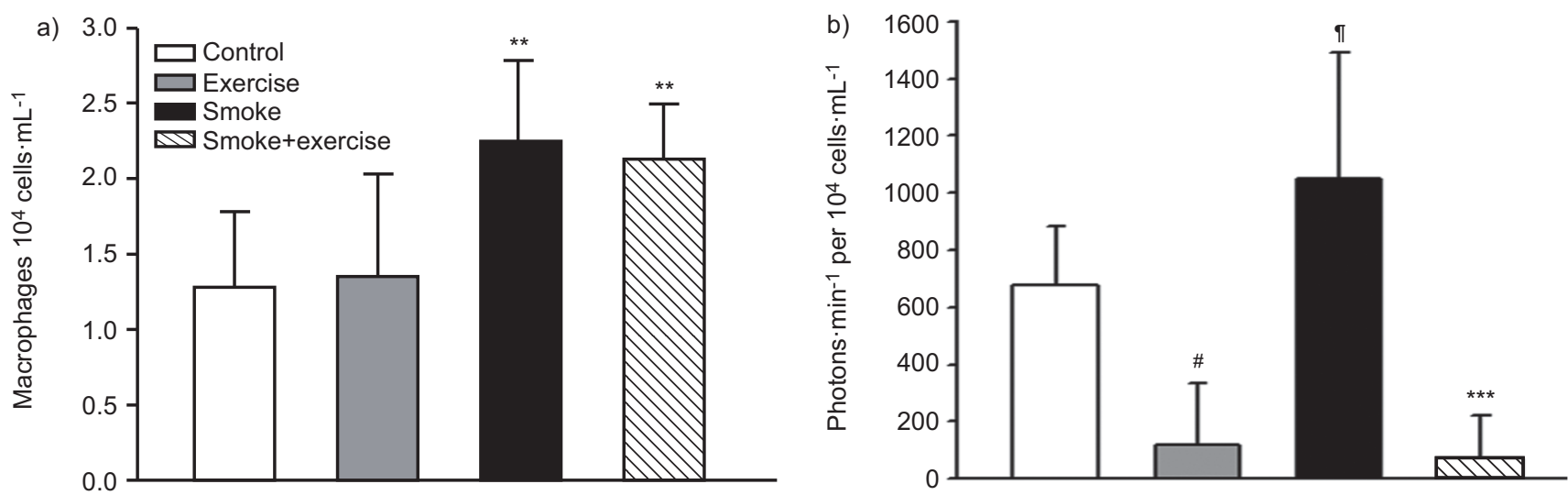

FIGURE 4. a) Number of macrophages in bronchoalveolar lavage (BAL) fluid. b) Concentration of reactive oxygen species in BAL. Data are presented as means and SD. \#: $p=0.001$ compared with control; ${ }^{*}: p=0.029$ compared with control; **: $p<0.01$ compared with the groups not exposed to cigarette smoke (control and exercise); ***: $p<0.001$ compared with smoke group.

because the physiological changes present in experimental models of COPD induced by exposure to cigarette smoke mimic a mild form of the disease in humans [22].

We used the mean linear intercept as a marker of the degree of alveolar distension, and our results are consistent with the development of emphysema, similar to previous studies [21, 23]. We observed enlargement of airspaces in the two lung compartments, defined by the distance from the pleura to the airways, suggesting that the lesion that develops in the lung in this experimental model is diffuse.

In our protocol, the effects of cigarette smoke exposure were more intense in pulmonary parenchyma than in the airways. In fact, we did not observe a significant difference in Raw values between the control group and mice exposed to cigarette smoke and we did not observe an increase in the density of inflammatory cells in the airways of mice exposed to cigarette smoke (data not shown). In contrast, we found a decrease in $\mathrm{H}$, suggesting a decrease of elastic recoil capacity, compatible with the functional profile of human pulmonary emphysema, and a decrease in G. Our protocol of aerobic physical training protected the mice from the decrease in $\mathrm{H}$ observed in mice exposed to cigarette smoke, which was probably secondary to the inhibition of alveolar wall destruction by exercise. In contrast, we did not observe an effect of aerobic training on G. Tissue resistance may be influenced by the amount of collagen fibres in alveolar walls, and aerobic conditioning did not have an effect on the amount of alveolar wall collagen. Collagen fibres are the most abundant fibres in alveolar walls and perhaps most critical for the mechanical properties of lung parenchyma [24]. Interestingly, in a protocol of short-term (2-month) exposure to cigarette smoke, MENEGALI et al. [21] did not observe an effect of daily swimming on the amount of proline in the lungs of C57BL/ 6 mice.
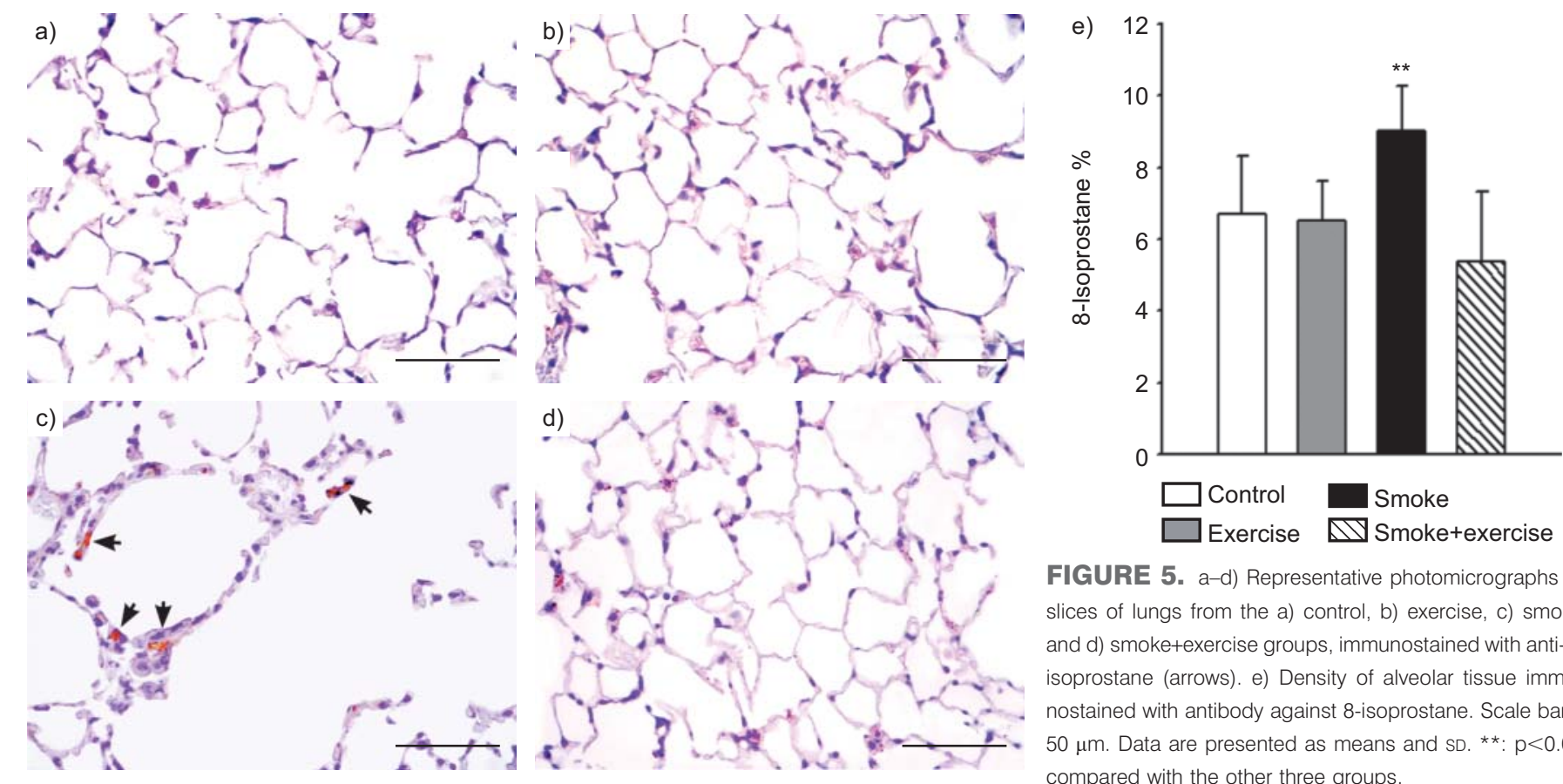

FIGURE 5. a-d) Representative photomicrographs of slices of lungs from the a) control, b) exercise, c) smoke and d) smoke+exercise groups, immunostained with anti-8isoprostane (arrows). e) Density of alveolar tissue immunostained with antibody against 8-isoprostane. Scale bars: $50 \mu \mathrm{m}$. Data are presented as means and SD. **: $p<0.01$ compared with the other three groups. 

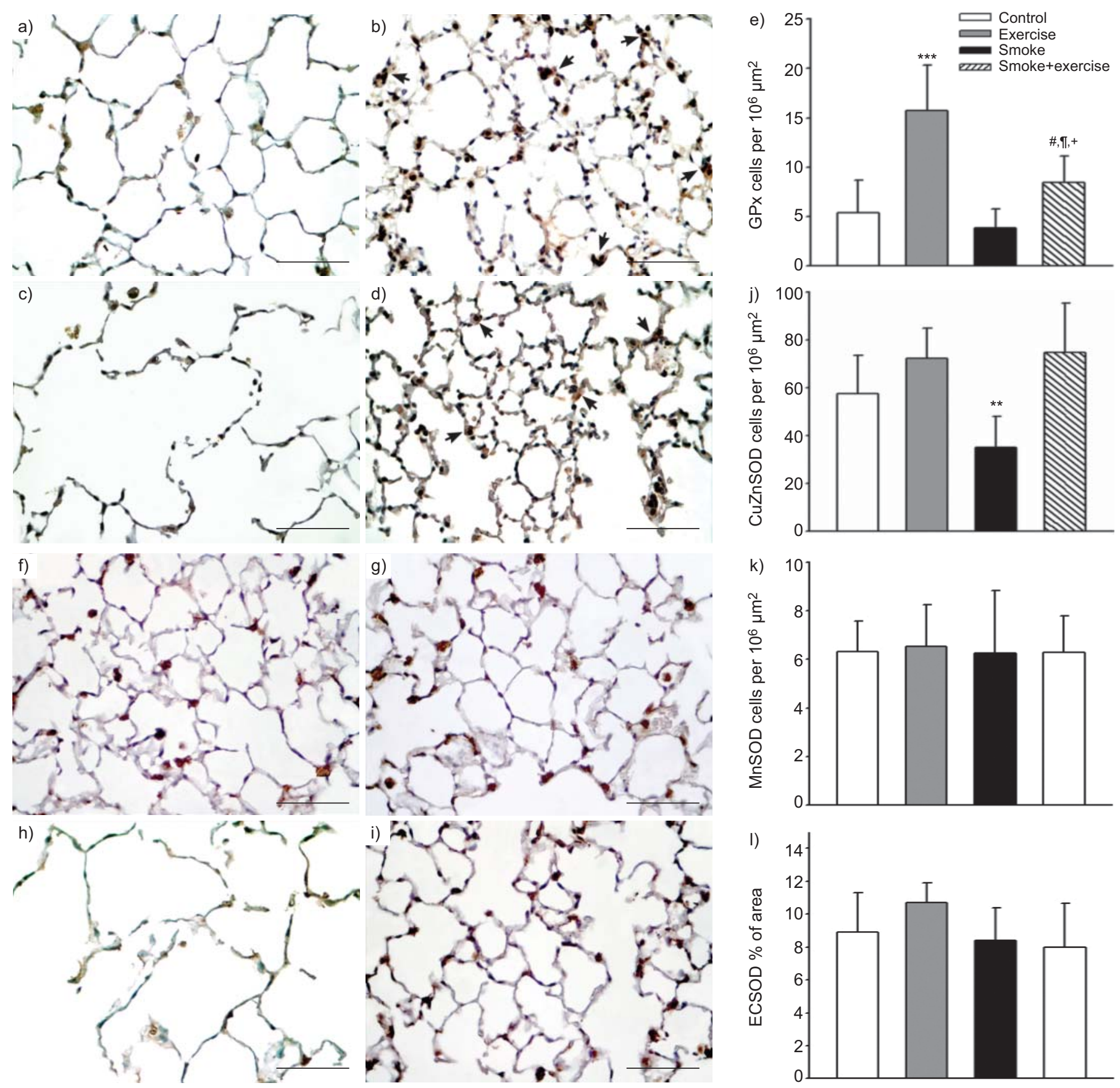

FIGURE 6. a-d) Representative photomicrographs of slices of lungs from a) control, b) exercise, c) smoke and d) smoke+exercise groups, immunostained with antiglutathione peroxidase (anti-GPx; arrows). e) Density of cells expressing GPx in alveolar walls. $\mathrm{f}-\mathrm{i}$ ) Representative photomicrographs of slices of lungs from f) control, g) exercise, h) smoke and i) smoke+exercise groups, immunostained with anti-CuZn superoxide dismutase (anti-CuZnSOD). j) Density of cells in alveolar walls positive for CuZnSOD. $k$ and I) Density of cells in alveolar walls positive for k) MnSOD and I) extracellular SOD (ECSOD). Scale bars: $50 \mu \mathrm{m}$. Data are presented as means and SD. ${ }^{*}: p<0.001$ compared with exercise group; ${ }^{\prime}: p=0.008$ compared with smoke group; ${ }^{+}: p=0.046$ compared with control group; **: $p<0.01$ compared with the other three groups; ***: $p<0.001$ compared with control and smoke groups.

Although the pathogenesis of COPD remains incompletely understood, oxidant-antioxidant and protease-antiprotease imbalances and pulmonary inflammation are central processes associated with the development of this disease, but the participation of each of these mechanisms and how they interact are still unclear [7]. Previous studies have shown that chronic exercise training induces the activity of some antioxidant enzymes [25] and anti-inflammatory mediators [26].

We observed an increase in the total number of inflammatory cells and macrophages in BAL of mice exposed to cigarette smoke, corroborating previous studies [27]. The macrophages 

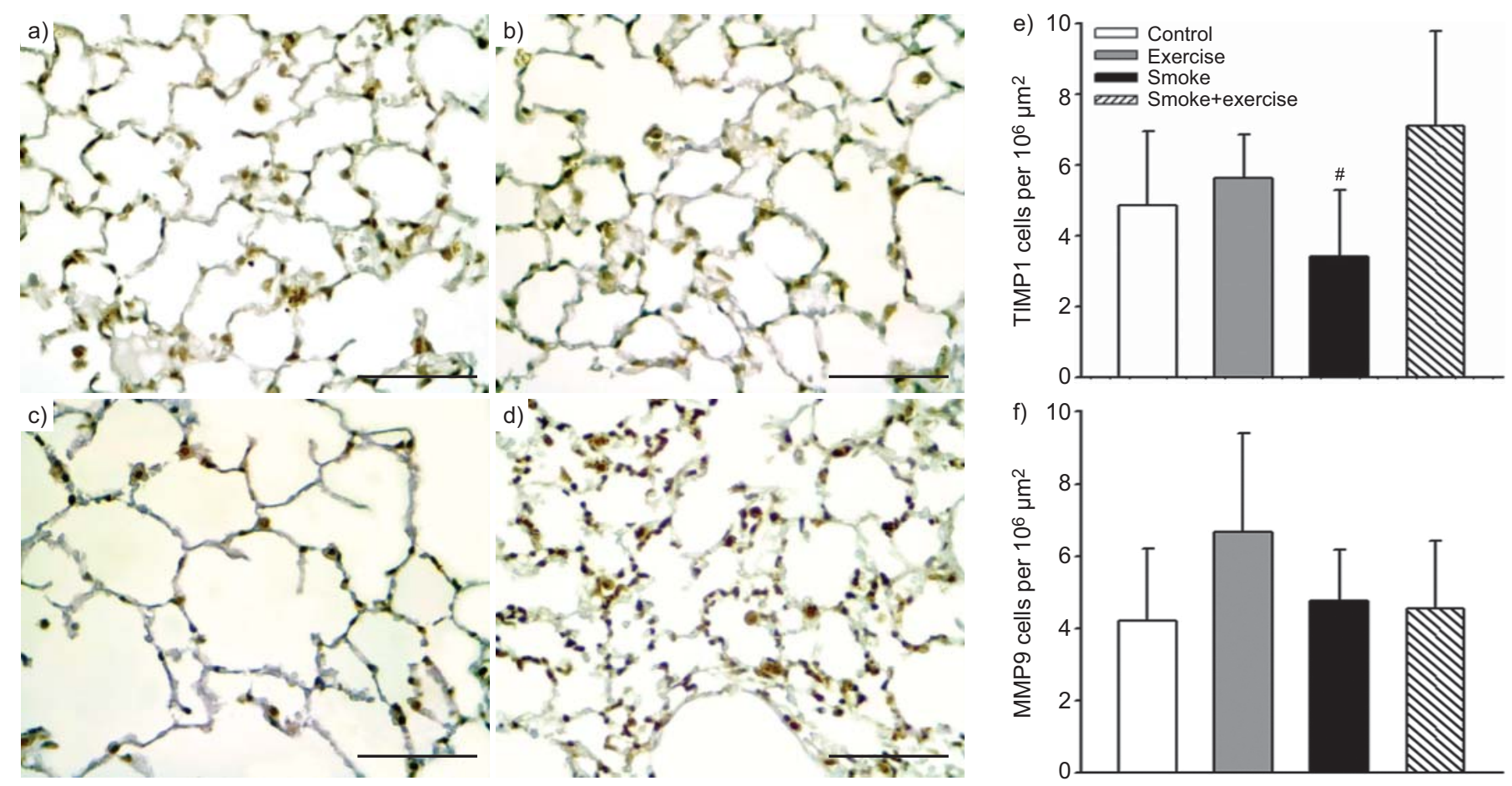

FIGURE 7. a-d) Representative photomicrographs of slices of lungs from the a) control, b) exercise, c) smoke and d) smoke+exercise groups, immunostained with anti-tissue inhibitor of metalloproteinase 1 (anti-TIMP1). e) Density of cells in alveolar walls positive for TIMP1. f) Density of cells in alveolar walls positive for matrix metalloproteinase (MMP)9. Scale bars: $50 \mu \mathrm{m}$. Data are presented as means and SD. ${ }^{\#}: p<0.02$ compared with the other three groups.

induced by substances in cigarettes may also secrete ROS and a chemotactic peptide for monocytes (MCP1) [28]. In our study, we observed a significant increase in MCP1 in mice exposed to cigarette smoke as described previously in BAL of smokers [29]. It was previously shown that some of the anti-inflammatory effects of physical training occur by reducing the levels of MCP1 [30, 31]. Despite the fact that exercise training did not modify the quantities of macrophages in BAL of mice exposed to cigarette smoke in our study, exercise reduced the expression of MCP1 by inflammatory cells in lung parenchyma induced by exposure to cigarette smoke. However, exercise training substantially inhibited ROS production in BAL of both control mice and mice exposed to cigarette smoke. In addition, the density of 8isoprostane in lung parenchyma was increased in mice exposed to cigarette smoke, and exercise training inhibited this increase. Isoprostanes are prostaglandin-like compounds that are formed from the peroxidation of arachidonic acid and are considered accurate markers of oxidative stress in vivo. There is an increase in 8-isoprostanes in the urine of COPD patients [32].

It is possible that the decrease in ROS production in BAL and in the expression of 8-isoprostanes in pulmonary tissue were due to an increase in the activity of antioxidant enzymes. In fact, we observed an increase in the density of cells expressing the antioxidant enzyme GPx in the lungs of mice submitted to the exercise training protocol. Exercise training also reversed the decrease in the density of lung cells expressing CuZnSOD induced by cigarette smoke. GPx has a role in the protection against lipid peroxidation due to decomposition of lipid hydroperoxides [33], and SODs are the primary class of enzymes that initiate the process of detoxifying superoxide anions by converting them into hydrogen peroxide. It was previously shown that both GPx and SOD increase in skeletal muscle after exercise training [25].

In our study, exposure to cigarette smoke did not change the expression of GPx in the lung. Other studies did not show any appreciable changes in the GPx activity in lung tissue obtained from chronic smokers [34]. In contrast, in an experimental model of exposure to cigarette smoke, GPx activity was increased [35].

Aerobic exercise inhibited the reduction of $\mathrm{CuZnSOD}$ found in mice exposed to smoke, but the expression of ECSOD and MnSOD was not different in the four experimental groups. There are three mammalian SODs: SOD1, CuZnSOD, is located mainly in the cytosol of cells; mitochondrial MnSOD, or SOD2, is predominantly located in the mitochondria and the cytosol; and SOD3, or ECSOD, is present outside the cells, where it is bound to matrix proteins such as collagen [36]. Other studies showed a reduction in the total antioxidant activity of CuZnSOD in the airway epithelium of asthmatics [37] and an increase in the production of superoxide anions in these patients [38]. Despite the fact that excessive production of ROS, particularly superoxide anions, is implicated in the pathophysiology of COPD [7], we are not aware of previous reports showing the expression of CuZnSOD in this disease.

RusH et al. [39] observed an elevation of CuZnSOD in the aortic endothelium of trained guinea pigs, and, although the protein levels of MnSOD were not altered, the levels of p67phox, a subunit of the enzyme pro-oxidant NADPH (nicotinamide adenine dinucleotide phosphate) oxidase, was reduced by exercise. 

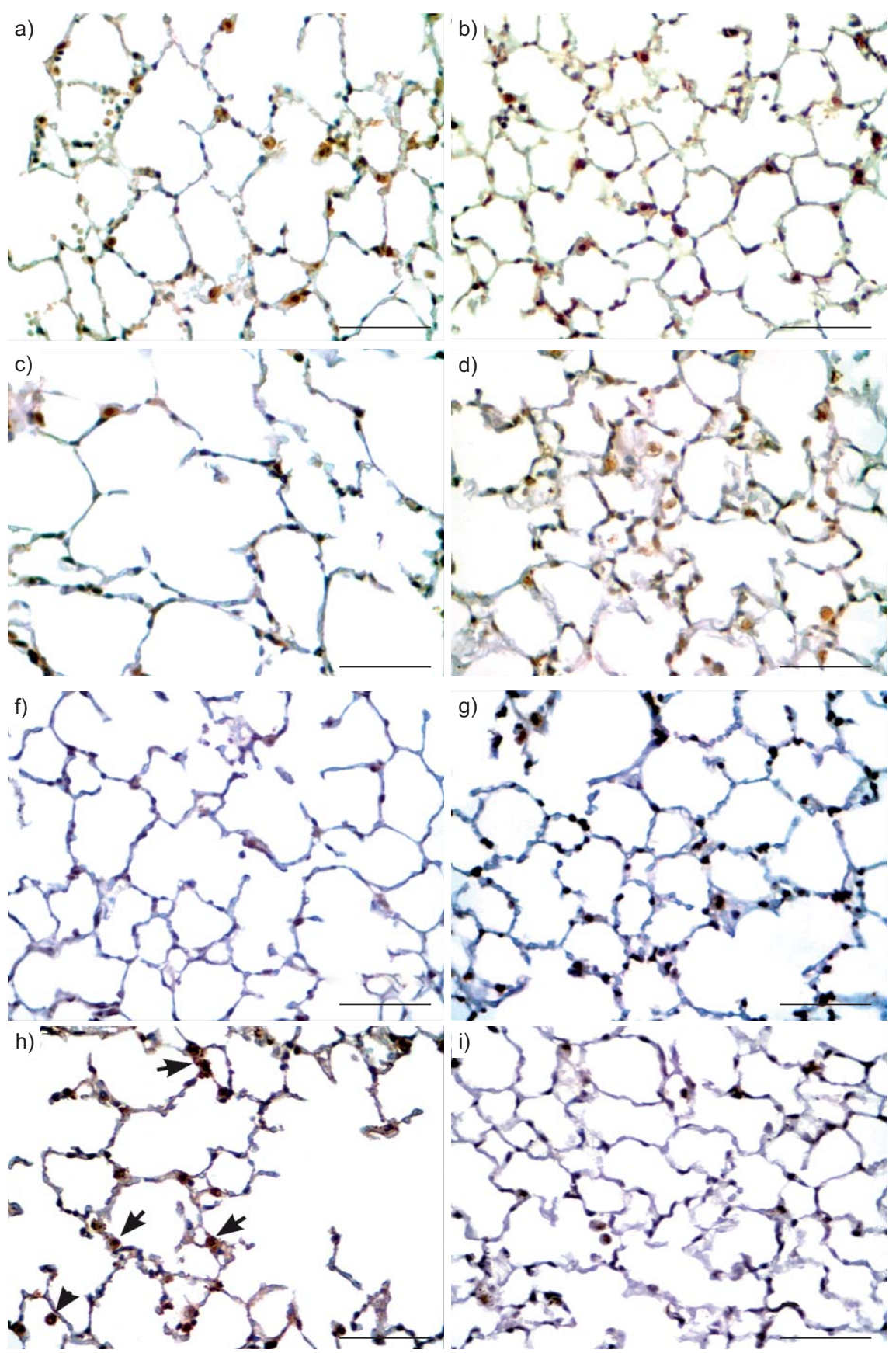
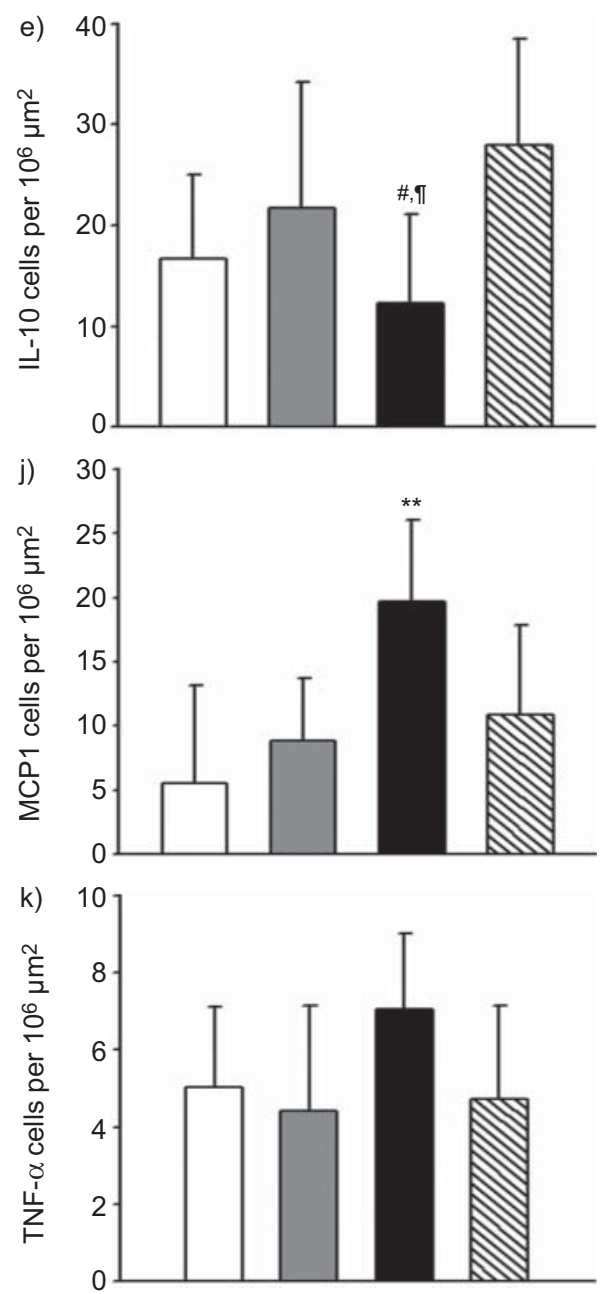

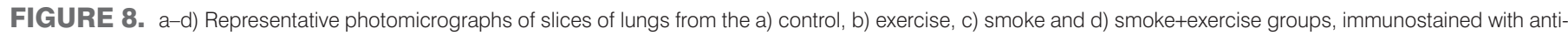

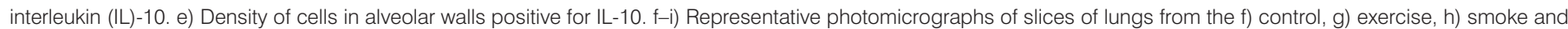

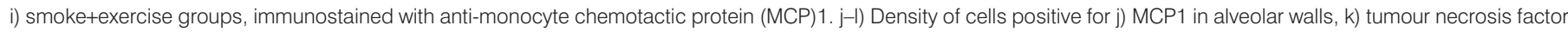

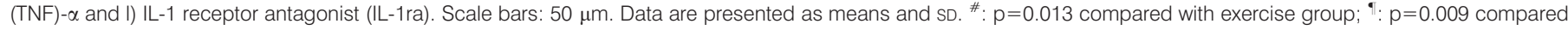
with smoke+exercise group; **: $\mathrm{p}<0.01$ compared with the other three groups.

Previous studies have shown that aerobic exercise can produce anti-inflammatory effects against allergic lung inflammation, and part of these effects are mediated by the release of the antiinflammatory cytokines IL-10 and IL-1ra, and also by direct regulation of the release of IL-1 $\beta$ and tumour necrosis factor- $\alpha[10,40]$. The expression of IL-10, a potent anti-inflammatory cytokine, was reduced in our mice exposed to cigarette smoke, in contrast to the results of GUERASSIMOV et al. [19]. In our study, this smoke-induced reduction was inhibited by exercise training. IL-10 modulates the expression of some cytokines, soluble mediators and cell surface molecules by cells of myeloid origin, influencing the ability to activate and sustain immune and inflammatory responses [41].

Inhalation of cigarette smoke can also lead to the secretion of MMP2, MMP9, MMP12, neutrophil elastase and cathepsins K, L and $\mathrm{S}$ by macrophages. Some studies suggest that pro-inflammatory stimuli induced by smoking may regulate the activity of MMP9 in COPD [42]. However, in our mice, the expression of 
MMP9 was not increased by exposure to smoke, but we found a decrease in TIMP1 in mice exposed to cigarette smoke that was reversed by aerobic exercise. A decrease in the release of TIMPs by alveolar macrophages in COPD has also been seen by HIRANO et al. [43]. Some studies have shown that IL-10 suppresses the release of MMP9 from monocytes of patients with COPD and, at the same time, stimulates the release of its major endogenous inhibitor, TIMP1 [44]. It is likely that the imbalance between proteases and their macrophage antiproteases has a pathogenic role in our experimental model of emphysema.

Our study has some limitations. We did not evaluate the timecourse of the development of pulmonary effects of cigarette smoke and the influence of exercise on the oxidant-antioxidant balance during the emphysema development. In addition, we did not evaluate the effects of both cigarette smoke and aerobic training on the skeletal muscles. Future studies should be performed to clarify these points.

Many benefits of exercise training in COPD patients have been previously shown. Exercise alleviates symptoms, improves quality of life, and decreases exacerbation episodes and the risk of respiratory mortality in COPD. Our study suggests that exercise training may be important not only in pulmonary rehabilitation in COPD but also as an adjuvant in the prevention and progression of pulmonary disease due to cigarette smoking.

In summary, we have shown that regular aerobic physical training of moderate intensity reduces oxidative stress and protects against the development of emphysema in mice. Exercise training inhibited the emphysema development by a significant decrease in oxidative stress, with reductions in ROS production in BAL and in 8-isoprostane expression in lung parenchyma, and with increases in antioxidant enzymes (GPx and CuZnSOD), TIMP1 and IL-10 in the inflammatory cells of the alveolar wall. These results support the hypothesis that the antioxidant effect of exercise combats cellular oxidative stress, which is induced by cigarette smoke and has an important role in COPD development.

\section{SUPPORT STATEMENT}

This work was supported by the following Brazilian scientific agencies: Fundação de Amparo à Pesquisa do Estado de São Paulo (FAPESP), Conselho Nacional de Desenvolvimento Científico e Tecnológico (CNPq), Laboratórios de Investigação Médica da Faculdade de Medicina da Universidade de São Paulo (LIMHC-FMUSP) and Coordenação de Aperfeiçoamento de Pessoal de Nível Superior (CAPES).

\section{STATEMENT OF INTEREST}

None declared.

\section{REFERENCES}

1 Menezes AM, Perez-Padilla R, Jardim JR, et al. Chronic obstructive pulmonary disease in five Latin American cities (the PLATINO study): a prevalence study. Lancet 2005; 366: 1875-1881.

2 Garcia-Aymerich J, Lange P, Benet M, et al. Regular physical activity modifies smoking-related lung function decline and reduces risk of chronic obstructive pulmonary disease: a population-based cohort study. Am J Respir Crit Care Med 2007; 175: 458-463.

3 Radak Z, Naito H, Kaneko T, et al. Exercise training decreases DNA damage and increases DNA repair and resistance against oxidative stress of proteins in aged rat skeletal muscle. Pflugers Arch 2002; 445: 273-278.
4 Radak Z, Chung HY, Goto S. Systemic adaptation to oxidative challenge induced by regular exercise. Free Radic Biol Med 2008; 44: 153-159.

5 Calabrese EJ, Baldwin LA. Hormesis: the dose-response revolution. Annu Rev Pharmacol Toxicol 2003; 43: 175-197.

6 Chapman KR, Mannino DM, Soriano JB, et al. Epidemiology and costs of chronic obstructive pulmonary disease. Eur Respir J 2006; 27: 188-207.

7 Cosio BG, Agustí A. Update in chronic obstructive pulmonary disease 2009. Am J Respir Crit Care Med 2010; 181: 655-660.

8 Church DF, Pryor WA. Free-radical chemistry of cigarette smoke and its toxicological implications. Environ Health Perspect 1985; 64: 111-126.

9 Frei B, Forte TM, Ames BN, et al. Gas phase oxidants of cigarette smoke induce lipid peroxidation and changes in lipoprotein properties in human blood plasma. Protective effects of ascorbic acid. Biochem J 1991; 277: 133-138.

10 Vieira RP, Claudino RC, Duarte AC, et al. Aerobic exercise decreases chronic allergic lung inflammation and airway remodeling in mice. Am J Respir Crit Care Med 2007; 176: 871-877.

11 Hantos Z, Daroczy B, Suki B, et al. Input impedance and peripheral inhomogeneity of dog lungs. J Appl Physiol 1992; 72: 168-178.

12 Gomes RF, Shen X, Ramchandani R, et al. Comparative respiratory system mechanics in rodents. J Appl Physiol 2000; 89: 908-916.

13 Athayde KS, Cocuzza M, Agarwal A, et al. Development of normal reference values for seminal reactive oxygen species and their correlation with leukocytes and semen parameters in a fertile population. J Androl 2007; 28: 613-620.

14 Ramos DS, Olivo CR, Quirino Santos Lopes FD, et al. Lowintensity swimming training partially inhibits lipopolysaccharideinduced acute lung injury. Med Sci Sports Exerc 2010; 42: 113-119.

15 Takubo Y, Guerassimov A, Ghezzo H, et al. $\alpha_{1}$-antitrypsin determines the pattern of emphysema and function in tobacco smoke-exposed mice: parallels with human diseases. Am J Respir Crit Care Med 2002; 166: 1596-1603.

16 Dolhnikoff M, Mauad T, Ludwig MS. Extracellular matrix and oscillatory mechanics of rat lung parenchyma in bleomycininduced fibrosis. Am J Respir Crit Care Med 1999; 160: 1750-1757.

17 Simões SM, Santos MA, Oliveira MS, et al. Inflammatory cell mapping of the respiratory tract in fatal asthma. Clin Exp Allergy 2005; 35: 602-611.

18 Wright JL, Cosio M, Churg A. Animal models of chronic obstructive pulmonary disease. Am J Physiol Lung Cell Mol Physiol 2008; 295: L1-L15.

19 Guerassimov A, Hoshino Y, Takubo Y, et al. The development of emphysema in cigarette smoke-exposed mice is strain dependent. Am J Respir Crit Care Med 2004; 170: 974-980.

20 Bartalesi B, Cavarra E, Fineschi S, et al. Different lung responses to cigarette smoke in two strains of mice sensitive to oxidants. Eur Respir J 2005; 25: 15-22.

21 Menegali BT, Nesi RT, Souza PS, et al. The effects of physical exercise on the cigarette smoke-induced pulmonary oxidative response. Pulm Pharmacol Ther 2009; 22: 567-573.

22 Rabe KF, Hurd S, Anzueto A, et al. Global strategy for the diagnosis, management, and prevention of chronic obstructive pulmonary disease: GOLD executive summary. Am J Respir Crit Care Med 2007; 176: 532-555.

23 Kasagi S, Seyama K, Mori $\mathrm{H}$, et al. Tomato juice prevents senescence-accelerated mouse P1 strain from developing emphysema induced by chronic exposure to tobacco smoke. Am J Physiol Lung Cell Mol Physiol 2006; 290: L396-L404.

24 Suki B, Ito S, Stamenovic D, et al. Biomechanics of the lung parenchyma: critical roles of collagen and mechanical forces. J Appl Physiol 2005; 98: 1892-1899.

25 Ji LL. Modulation of skeletal muscle antioxidant defense by exercise: role of redox signaling. Free Radic Biol Med 2008; 44: 142-152. 
26 Petersen AM, Pedersen BK. The anti-inflammatory effect of exercise. J Appl Physiol 2005; 98: 1154-1162.

27 Finkelstein R, Fraser RS, Ghezzo H, et al. Alveolar inflammation and its relation to emphysema in smokers. Am J Respir Crit Care Med 1995; 152: 1666-1672.

28 Barnes PJ, Shapiro SD, Pauwels RA. Chronic obstructive pulmonary disease: molecular and cellular mechanisms. Eur Respir J 2003; 22: 672-688.

29 Capelli A, Di Stefano A, Gnemmi I, et al. Increased MCP-1 and MIP-1 $\beta$ in bronchoalveolar lavage fluid of chronic bronchitics. Eur Respir J 1999; 14: 160-165.

30 Trøseid M, Lappegård KT, Claudi T, et al. Exercise reduces plasma levels of the chemokines MCP-1 and IL- 8 in subjects with the metabolic syndrome. Eur Heart J 2004; 25: 349-355.

31 Adamopoulos S, Parissis J, Kroupis C, et al. Physical training reduces peripheral markers of inflammation in patients with chronic heart failure. Eur Heart J 2001; 22: 791-797.

32 Pratico D, Basili S, Vieri M, et al. Chronic obstructive pulmonary disease is associated with an increase in urinary levels of isoprostane F2 $\alpha$-III, an index of oxidant stress. Am J Respir Crit Care Med 1998; 158: 1709-1714.

33 Federico A, Morgillo F, Tuccillo C, et al. Chronic inflammation and oxidative stress in human carcinogenesis. Int J Cancer 2007; 121 : 2381-2386.

34 Pasquini R, Sforzolini GS, Cavaliere A, et al. Enzymatic activities of human lung tissue: relationship with smoking habits. Carcinogenesis 1988; 9: 1411-1416.
35 York GK, Peirce TH, Schwartz LW, et al. Stimulation by cigarette smoke of glutathione peroxidase system enzyme activities in rat lung. Arch Environ Health 1976; 31: 286-290.

36 Kinnula VL, Crapo JD. Superoxide dismutases in the lung and human lung diseases. Am J Respir Crit Care Med 2003; 167: 1600-1619.

37 Smith LJ, Shamsuddin M, Sporn PH, et al. Reduced superoxide dismutase in lung cells of patients with asthma. Free Radic Biol Med 1997; 22: 1301-1307.

38 Jarjour NN, Busse WW, Calhoun WJ. Enhanced production of oxygen radicals in nocturnal asthma. Am Rev Respir Dis 1992; 146: 905-911.

39 Rush JW, Turk JR, Laughlin MH. Exercise training regulates SOD-1 and oxidative stress in porcine aortic endothelium. Am J Physiol Heart Circ Physiol 2003; 284: H1378-H1387.

40 Chen HI, Hsieh SY, Yang FL, et al. Exercise training attenuates septic responses in conscious rats. Med Sci Sports Exerc 2007; 39: 435-442.

41 Moore KW, de Waal Malefyt R, Coffman RL, et al. Interleukin-10 and the interleukin-10 receptor. Annu Rev Immunol 2001; 19: 683-765.

42 Finlay GA, O'Driscoll LR, Russell KJ, et al. Matrix metalloproteinase expression and production by alveolar macrophages in emphysema. Am J Respir Crit Care Med 1997; 156: 240-247.

43 Hirano K, Sakamoto T, Uchida Y, et al. Tissue inhibitor of metalloproteinases-2 gene polymorphisms in chronic obstructive pulmonary disease. Eur Respir J 2001; 18: 748-752.

44 Lacraz S, Nicod LP, Chicheportiche R, et al. IL10 inhibits metalloproteinase and stimulates TIMP1 production in human mononuclear phagocytes. J Clin Invest 1995; 96: 2304-2310. 